\title{
Modelling FRR of Biometric Verification Systems Using the Template Co-update Algorithm
}

\author{
Luca Didaci, Gian Luca Marcialis, and Fabio Roli \\ Dept. of Electrical and Electronic Eng., University of Cagliari, Italy \\ \{luca.didaci, marcialis, roli\}@diee.unica.it
}

\begin{abstract}
The decrease of representativeness of available templates during time is due to the large intra-class variations characterizing biometrics (e.g. faces). This requires the design of algorithms able to make biometric verification systems adaptive to such variations. Among others, the template co-update algorithm, which uses the mutual help of two complementary biometric matchers, has shown promising experimental results. The present paper is aimed to describe a theoretical model able to explain the co-update behaviour. In particular, the focus is on the relationships between error rate and gallery size increase. Preliminary experimental results are shown to validate the proposed model.
\end{abstract}

\section{Introduction}

Personal verification systems based on biometrics reached very notable improvements in the last years [1-2]. In particular, multi-modal verification systems allow to obtain an increase of performance by exploiting advantages of two or more biometrics.

However, many open issues still remain. Among others, the dependency of performance from the biometric variations related to short-time or medium-time causes. For example, a scratch on the finger skin or the face, or an unknown expression, or the so-called "aging" effect, which is crucial in performance of face recognition systems [1]. With regard to this problem, the topic called "template update" has received an increasing interest. The template(s) is a sample, or a set of samples, which is considered "representative" of the subject appearance [1]. Templates are usually captured during the enrolment session, under the supervision of a human expert. However, it is widely acknowledged that capturing all possible subject variations during an enrolment session is impossible. On the other hand, the system performance strongly depends on such representativeness. Accordingly, updating a template, by additional enrolment sessions, is considered as a good solution in order to follow the intra-class variations of each biometric [3].

Recently, "semi-supervised" [4-9] template update has been proposed. In this approach, unlabelled samples (that is, collected during system operations) showing very strong similarity to existing templates are added into the gallery of the related subject, thus increasing the representativeness of related set of templates. Template update solutions involve both mono-modal and multi-modal verification systems [3-10]. In particular, the so-called "co-update" algorithm showed that the mutual "help" of two weakly correlated biometrics (e.g., fingerprints and faces) can increase the gallery 
representativeness more quickly than systems adopting only one biometric [7-8], thus improving the overall performance.

In [10], a statistical framework modelling the co-update behaviour has been proposed. This model starts from hypotheses which fit the practical scenarios involving fingerprint and face biometrics, providing a first analytical framework able to explain the relationship between the amount of unlabelled data collected and the gallery size for a certain application. However, that model is lack of a convincing explanation of the verification performance of the system, in particular, the False Rejection Rate, when the estimated FAR is zero. Moreover, that model underestimates the FRR, especially in case of clients not "easily" recognizable, as we show in this paper. The aim of the present work is to fill such gap by adopting a more realistic model for genuine users distribution. This allows to point out the relationships between the amount of unlabelled data collected and the error rate reduction achieved. The impact is practical, since this model can be adopted by designers for the preliminary assessment of template co-update algorithms in real environments. Experimental results on a chimerical data set show the reliability of the framework by comparing the gallery sizes and errors predicted by the model with the gallery sizes and errors obtained on test data.

The paper is organized as follows. Section 2 describes the current version of the model. Section 3 describe our proposal for the modelling of verification performance as function of the gallery size increase. Section 4 reports the experimental results which validate the model, and also show the difference with the previous one. Section 5 concludes the paper.

\section{Recent Advances in Modelling the Template Co-update Algorithm}

The "complementary" performances of biometric matchers using distinct biometric traits, for example, face and fingerprint, are one of the fundamental motivations for multi-modal biometrics [7,11]. Intuitively, each recognizer is expected to assign correct labels to certain input data which are difficult for the other. In the case of template co-update, two matchers are used (bi-modal system).

Description of co-update algorithms is given in Figure 1. Let $D_{l}$ be a set of labelled biometric data. These data are the templates enrolled by human supervision, thus they are labelled with users identities (clients names). It is supposed that a batch $D_{u}$ (usually, much larger than $D_{l}$ ) of data is acquired during system operations. Each element of $D_{u}$ is a pair of biometric samples, i.e. face and fingerprint, from the same individual. During the off-line co-update phase, each matcher is applied to the batch $D_{u}$ to verify the identity of each couple of biometric samples. A biometric sample is added to the set $D_{l}$ if and only if the identity of the other biometric sample of the couple has been verified.

In this paper, we adopted the same terminology used in [10] for sake of uniformity with the state-of-the-art. Let us call "master" the biometric that assumes the supervisor's role, and "slave" the biometric whose gallery is augmented thanks to the master biometric. During the co-training process both biometrics assume, alternatively, master and slave roles. The process is repeated a specified number of times. 
As for the majority of semi-supervised template update algorithms, the core of coupdate is the insertion of new samples without external supervision. This might introduce misclassified samples in gallery. For this reason, only "confident examples" are added by a very high co-update threshold value at which no false matches occur, namely, FAR $=0 \%$.

Co-update pros and cons have been preliminary evaluated in [8]. But only in [10] a first trial to theoretically explain why it works has been made. In the following, we summarize the main achievements of framework proposed in Ref. [10], and in Section 3 we specify what we added in order to improve that framework.

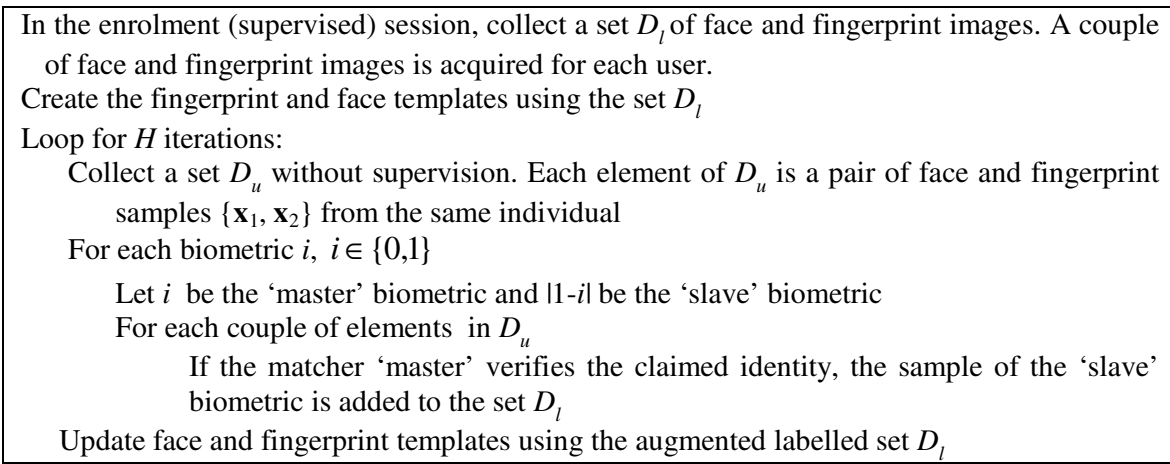

Fig. 1. Co-updating algorithm. The main steps of the algorithm are shown.

Let $n$ be the number of different templates contained in a gallery, for a given identity. Let $N_{T O T}$ be the maximum number of templates that a gallery can contain. The role, master or slave, of the two biometrics, is highlighted by superscripts $\mathrm{M}$ and $\mathrm{S}$.

Some assumptions are made in order to propose a mathematically tractable model [10]. The first assumption regards the finite discrete space used to represent different biometric impressions. Each identity can produce a maximum of $N_{T O T}^{(\text {face })}$ and $N_{\text {TOT }}^{\text {(fingerprint) }}$ different observations of the face and fingerprint biometrics, respectively. This assumption can be motivated easily in strongly controlled environments, where a small amount of substantial intra-class variations is admitted, but not collectable by the supervised enrolling. The main consequence of this assumption is that a new example, very similar to an existing one, can be considered already present in the gallery.

The second assumption states that all biometric impressions are equally probable (as usually stated in biometric applications), and the two traits are conditionally independent given the identity. The motivation is that no correlation is supposed to be between the face appearance and the related client's fingerprint image [1-2].

In the co-update stage, a set $D_{u}$ of $\left|D_{u}\right|=k$ pairs of biometric samples (i.e. face and fingerprint) are presented to the system. We indicate with $k$ ' the number of samples of the master biometric whose identity has been verified (i.e. is over the updating threshold in practice). 
The value $k$ ' can be easily computed by considering that each of $k$ biometric samples of the master biometric can be drawn with replacement from a homogeneous population of $N_{T O T}^{(M)}$ elements. The probability that a sample will be verified is $p=1-F R R^{(M)}$, where $F R R^{(M)}$ is the False Rejection Rate for the master biometric. The problem can be modeled using a binomial distribution [10]. Therefore, on average:

$$
k^{\prime}=k\left(1-F R R^{(M)}\right) .
$$

It is worth noting that the effectiveness of the master biometric as supervisor towards the 'slave' biometric is related to its FRR value. If $\mathrm{FRR}=0 \quad(k$ ' $=k)$ the master biometric is equivalent to a "true" supervisor because it will verify the claimed identity of all 'genuine' samples in $D_{u}$. If FRR $>0$, then only $k$ ' $<k$ samples can be verified. Thus, only $k^{\prime}<k$ samples will be added to the slave gallery.

Now, let $d$ be the random variable representing the number of elements in $k$ ' that are different among themselves, and $d n$ the random variable representing the number of elements in $k$ ' that are different and not present in gallery.

The pdf $p_{d}(d)$ can be modeled using a multinomial distribution:

$$
p_{d}(d)=\left(\begin{array}{c}
N_{T O T} \\
d
\end{array}\right) \sum_{i=0}^{d}(-1)^{i}\left(\begin{array}{c}
d \\
i
\end{array}\right)\left(\frac{d-i}{N_{T O T}}\right)^{k^{\prime}} .
$$

The conditional pdf $p_{d n / d}(d n / d)$ - the probability to have $d n$ different samples not present in gallery if $d$ samples are different among themselves - can be modeled using an hyper-geometric distribution:

$$
p_{d n l d}(d n \mid d)=h\left(d n ; N_{\text {TOT }},\left(N_{\text {TOT }}-n\right), d\right) .
$$

where $N_{\text {TOT }}$ is the number of items in the population, $\left(N_{\text {TOT }}-n\right)$ is the number of items in the population that are classified as 'highly confident genuine users', $d$ is the number of items in the sample, $d n$ is the number of items in the sample that are classified as 'highly confident genuine users'.

By using Eqs. (2) and (3), the pdf $p_{d n}(d n)$ is derived [10]. Here, we are interested to the expected value $E[d n]$. This represents the expected enlargement of the gallery due to the collection of $k$ ' elements. By assuming that for each co-update step the number $\Delta n^{(S)}$ of new and distinct samples added to the slave gallery is about equal to its expected value $E[d n]$ :

$$
\Delta n^{(S)} \approx E[d n]=\sum_{d n=0}^{k^{\prime}}\left\{d n \cdot p_{d n}(d n)\right\}=\left(1-\left(1-\frac{1}{N_{T O T}^{(S)}}\right)^{k\left(1-F R R^{(M)}\right)}\right)\left(N_{T O T}^{(S)}-n^{(S)}\right) .
$$

Let $n_{0}^{(S)}$ be the initial size of the gallery, and $n_{(i)}^{(S)}$ the size of the gallery at the $i$-th co-update step. The length of the gallery at the $i$-th step can be written as: 


$$
\begin{aligned}
& n_{(0)}^{(S)}=n_{0}^{(S)} \\
& n_{(i)}^{(S)}=n_{(i-1)}^{(S)} w+N_{T O T}^{(S)}(1-w) ; w=\left(1-1 / N_{T O T}^{(S)}\right)^{k\left(1-F R R^{(M)}\right)} .
\end{aligned}
$$

The weight $w$ takes into account the effectiveness of the master gallery as a supervisor.

The FRR $^{(\mathrm{M})}$ value determine the system's dynamics, so it is worth to relate its value to the length of the master gallery.

\section{The Problem of Modeling FRR}

In order to model FRR, in [10] has been introduced, for each client $i$, a parameter called "connection degree" $m_{i}$, correspondent to the average number of examples that produce a "score' over the updating threshold. Therefore, "difficult" clients can be modeled by a low value of $m_{i}$, whilst high value of $m_{i}$ is adopted for "easy" clients. In [10], the probability that no examples among the $m_{i}$ are in the gallery, which correspond to FRR value, has been computed:

$$
F R R^{(M)}= \begin{cases}0 & \text { if } m_{i}>\left(N_{T O T}^{(M)}-n^{(M)}\right. \\ h\left(r=0 ; N_{T O T}^{(M)}, m_{i}, n^{(M)}\right) & \text { if } m_{i} \leq\left(N_{T O T}^{(M)}-n^{(M)}\right)\end{cases}
$$

Where $h$ is a hypergeometric distribution on a random variable $r$ equal to the number of examples, in the gallery, among the $m_{i}$.

This approach is too rough, because it does not take into account that, actually, each sample has his own " $m_{i}$ ". In other words, each $\mathrm{j}$-th biometric sample is characterized by a $m_{i j} \geq 0$ connection degree with other samples, and the $i$-th client is characterized by the sequence $\left\{m_{i 1}, m_{i 2}, \ldots, m_{i N_{T O T}}\right\}$ of connection degrees. In particular, eq. (6) neglects that there is a subset of samples for which this connection degree is zero. We refer to such samples with the term "isolated". As a consequence, isolated samples contribute to the FRR with a constant value equal to 1 , because they cannot "match" to other samples.

Therefore, we propose to correct eq. (6) by considering the fraction of samples that are isolated $\left(m_{i j}=0\right)$, namely, $f_{I}$, and the fraction of connected samples $\left(m_{i j}>0\right), f_{C}$. Eq. (6) becomes as follows:

$$
F R R^{(M)}=\left\{\begin{array}{ll}
f_{I} \cdot 1+f_{C} \cdot 0 & \text { if } n^{(M)}>N_{T O T}^{(M)}-m_{i} \\
f_{I} \cdot 1+f_{C} \cdot h\left(0 ; N_{T O T}^{(M)}, m_{i}, n^{(M)}\right) & \text { if } n^{(M)} \leq N_{T O T}^{(M)}-m_{i}
\end{array} .\right.
$$

When the gallery reaches its maximum size, $n=N_{T O T}$, the FRR value depends only on the isolated samples, for the reason we explained above. In other words, there is a "saturation" value for FRR, that is $\operatorname{FRR}\left(n=N_{T O T}\right)=f_{I}$. It is worth noting that saturation value is 0 for the model according to eq. (6).

Eq. (5) allows us to model the gallery size increase during the co-update iterations, whilst eq. (7) models the FRR of the master biometrics in a more realistic manner than eq. (6). Notice that, in order to simplify the model, we adopted the average of $m_{i j}$ for connected samples. Now, this average value does not take into account isolated samples, but only connected samples. The above relationships can be used to predict the behaviour of co-update. In the next Section the experimental validation of the proposed model by eqs. $(5,7)$ is done by co-updating a face and a fingerprint matcher on real data. 


\section{Experimental Results}

\subsection{The Data Set}

The data set adopted consists of 42 individuals composed of 20 face and fingerprint images for each individual, by keeping in mind the independence of face and fingerprint traits. The time span of both the collected data sets spans over one year. Forty-two clients with 20 instances representing significant illuminations changes and variations in facial expressions per person were used from the Equinox corporation database [12]. The fingerprint data set has been collected by the authors using Biometrika Fx2000 optical sensor. The images are acquired with variations in pressure, moisture and time interval to represent large intra-class variations. The results are computed on five random coupling of face and fingerprint datasets and are averaged. Whilst minutiae are simply extracted from the fingerprint images after commonly used processing algorithms [2,13], PCA is computed on the whole data set and applied to face images in order to reduce the size of the overall feature space. $95 \%$ of energy is retained according to the current literature [1,14].

It is worth noting that the face data set is notably "simpler" than the fingerprint one. We adopted so different data sets in order to show the effect of intra-class variations on the model prediction ability.

\subsection{Experimental Protocol}

First of all, we implemented a simple bi-modal identification system made up of a PCA-based face matcher and a fingerprint matcher using the "String" matching algorithm ("String" is based on minutiae points). We used the standard versions of these two recognition algorithms [13-14]. Then, we implemented the template co-update algorithm in Figure 1. Both the eigenspace of the PCA-based face matcher and the coupdate threshold value at FAR $=0 \%$ are computed using the whole dataset, and has not been updated during the co-update process. This approach is common to some template update practices [4-5]. The update of face and fingerprint templates is performed simply by adding new examples to the user's gallery.

In the experiments the initial gallery has been set as follows. We selected, as the initial template in $D_{l}$ for the $i$-th client, a sample whose connection degree is exactly $m_{i}$. In other word, the initial template is a connected sample 'near' to other $m_{i}$ samples. In the proposed model the $i$-th client is characterized by $\left(f_{C}, m_{i}\right)$ so this initial template is representative for the client. The rationale for this choice is to exclude outliers from the initial gallery, likewise to what happens in real situations, where the initial template is chosen in completely supervised fashion.

We simulated the acquisition of a batch set $D_{u}$ by generating several sets of $k=10$ couples - face and fingerprint - of 'genuine' examples, drawn with replacement from a homogeneous population of $N_{T O T}^{(\text {face })}=N_{T O T}^{(\text {fingerprint })}=20$ samples. We are aware that adopted database size may not be very well appropriate for the task, but it respects, on average, the size adopted in other template update works reported in literature [4-9]. Since duplicated samples may provide high match scores, which is not realistic, we disregard the first highiest match score when such duplicates are present in the gallery. 
In order to set the correct parameters in the proposed model, for each client and for each biometric we computed a) the value $f_{c}$, that is, the fraction of samples that produce a 'score' over the threshold $S^{*}$, and b) the value $m_{i}$, that is, the integer nearest to the average connection degree of the connected samples. Table 1 shows the computed parameters. For each client, results are averaged on ten trials (experimental values) and predicted using parameters in Table 1.

Table 1. List of parameters used to model the chimerical dataset. Fraction of connected samples $\left(f_{\mathrm{c}}\right)$ and average connection degree characterizing the connected samples $\left(m_{i}\right)$ are reported for each client and for each biometric.

\begin{tabular}{|l|l|l|l|l|}
\hline \multirow{2}{*}{ ID } & \multicolumn{2}{|l|}{ face } & \multicolumn{2}{l|}{ fingerprint } \\
\cline { 2 - 5 } & $\mathrm{m}_{\mathrm{i}}$ & $\mathrm{f}_{\mathrm{c}}$ & $\mathrm{m}_{\mathrm{i}}$ & $\mathrm{f}_{\mathrm{c}}$ \\
\hline 1 & 1 & 0.4 & 0 & 0 \\
\hline 2 & 3 & 0.8 & 2 & 0.4 \\
\hline 3 & 3 & 0.85 & 2 & 0.2 \\
\hline 4 & 4 & 0.85 & 2 & 0.2 \\
\hline 5 & 3 & 0.75 & 1 & 0.25 \\
\hline 6 & 3 & 0.85 & 1 & 0.1 \\
\hline 7 & 2 & 0.65 & 1 & 0.2 \\
\hline 8 & 3 & 0.85 & 1 & 0.2 \\
\hline 9 & 2 & 0.65 & 0 & 0 \\
\hline 10 & 3 & 0.85 & 1 & 0.15 \\
\hline $\mathbf{1 1}$ & $\mathbf{4}$ & $\mathbf{1}$ & $\mathbf{1}$ & $\mathbf{0 . 3 5}$ \\
\hline 12 & 4 & 0.5 & 1 & 0.25 \\
\hline 13 & 3 & 0.9 & 1 & 0.35 \\
\hline 14 & 5 & 0.9 & 1 & 0.15 \\
\hline
\end{tabular}

\begin{tabular}{|l|l|l|l|l|}
\hline \multirow{2}{*}{ ID } & \multicolumn{2}{|l|}{ Face } & \multicolumn{2}{l|}{ fingerprint } \\
\cline { 2 - 5 } & $\mathrm{m}_{\mathrm{i}}$ & $\mathrm{f}_{\mathrm{c}}$ & $\mathrm{m}_{\mathrm{i}}$ & $\mathrm{f}_{\mathrm{c}}$ \\
\hline 15 & 3 & 0.65 & 0 & 0 \\
\hline 16 & 3 & 0.8 & 2 & 0.35 \\
\hline 17 & 2 & 0.55 & 1 & 0.3 \\
\hline 18 & 4 & 0.85 & 1 & 0.1 \\
\hline 19 & 2 & 0.75 & 3 & 0.3 \\
\hline 20 & 3 & 0.85 & 2 & 0.5 \\
\hline 21 & 3 & 0.75 & 1 & 0.1 \\
\hline 22 & 4 & 0.85 & 0 & 0 \\
\hline 23 & 4 & 0.8 & 1 & 0.15 \\
\hline 24 & 3 & 0.8 & 0 & 0 \\
\hline 25 & 4 & 0.75 & 2 & 0.45 \\
\hline 26 & 4 & 0.85 & 2 & 0.4 \\
\hline 27 & 3 & 0.9 & 1 & 0.35 \\
\hline 28 & 1 & 0.75 & 2 & 0.25 \\
\hline
\end{tabular}

\begin{tabular}{|l|l|l|l|l|}
\hline \multirow{2}{*}{ ID } & \multicolumn{2}{|l|}{ face } & \multicolumn{2}{l|}{ fingerprint } \\
\cline { 2 - 5 } & $\mathrm{m}_{\mathrm{i}}$ & $\mathrm{f}_{\mathrm{c}}$ & $\mathrm{m}_{\mathrm{i}}$ & $\mathrm{f}_{\mathrm{c}}$ \\
\hline 29 & 3 & 0.75 & 0 & 0 \\
\hline $\mathbf{3 0}$ & $\mathbf{2}$ & $\mathbf{0 . 6 5}$ & $\mathbf{1}$ & $\mathbf{0 . 2}$ \\
\hline 31 & 4 & 0.8 & 0 & 0 \\
\hline 32 & 2 & 0.35 & 3 & 0.2 \\
\hline 33 & 5 & 0.9 & 1 & 0.15 \\
\hline 34 & 2 & 0.6 & 1 & 0.1 \\
\hline 35 & 2 & 0.95 & 2 & 0.2 \\
\hline 36 & 3 & 0.85 & 0 & 0 \\
\hline 37 & 4 & 0.75 & 1 & 0.1 \\
\hline 38 & 4 & 0.85 & 0 & 0 \\
\hline 39 & 5 & 0.9 & 1 & 0.15 \\
\hline 40 & 3 & 0.8 & 0 & 0 \\
\hline 41 & 3 & 0.8 & 2 & 0.4 \\
\hline 42 & 2 & 0.65 & 1 & 0.1 \\
\hline
\end{tabular}

\subsection{Experimental Results}

FRR values and gallery sizes obtained on test data are compared to correspondent values by eqs. (5-7). Fig. 2 compares the values obtained on test data with the values predicted by the model proposed in [10] and by ours. Reported trend is obtained by averaging values over all the clients. For each iteration of the algorithm depicted in Fig. 1, the FRR value evaluated at zeroFAR and the gallery sizes are reported, whilst theoretical lines follow eqs. $(5,6)$ and eqs $(5,7)$. In order to highlight the peculiarity of the model, values regarding two typical clients, 'easy' and 'difficult', are also show in Figs. 3 (client 11) and 4 (client 30).

As we can deduce from Table 1, client 11 is an 'easy' client. The face biometric presents $m_{11}=4, f_{c}=1$. This means that every sample is connected, thus producing a score over the threshold $S^{*}$ with at least another sample. Being $m_{11}=4$, each sample is averagely connected to other four ones. Worth noting, the fingerprint biometric presents $m_{11}=1, f_{c}=0.35$ : only $35 \%$ of the samples are connected, with connection degree $m=1$. Remaining samples are isolated. They can be introduced in the gallery thanks to the master, face, biometric only. However, they don't contribute to the expressive power of the fingerprints gallery, since they cannot match with other samples.

Referring to the same Table 1, client 30 is a difficult client. The face biometric presents $m=2, f_{c}=0.65$ : only $65 \%$ of the samples are connected, with connection degree $m=2$. Moreover, the fingerprint biometric exhibits $m=1, f_{c}=0.2$. Other face and fingerprint samples are isolated, and, even if they are in the gallery, cannot contribute to its expressive power. 


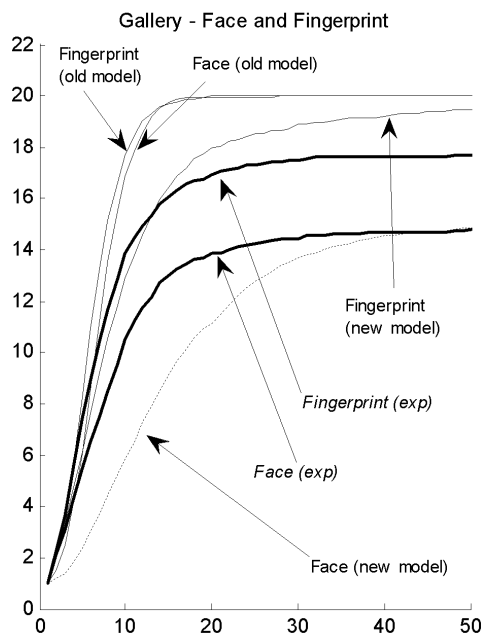

(a)

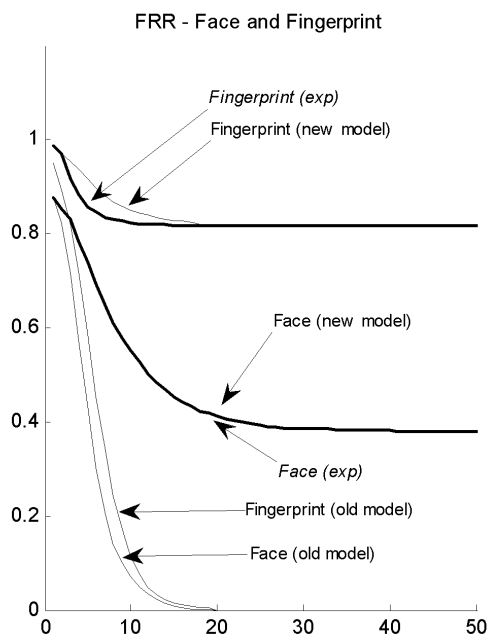

(b)

Fig. 2. Experimental trend and predicted values of gallery sizes (a) and FRR (b) for fingerprint and face biometrics. Reported trend is obtained averaging values over all the clients. Values predicted by the previous model and the new proposed model are shown.

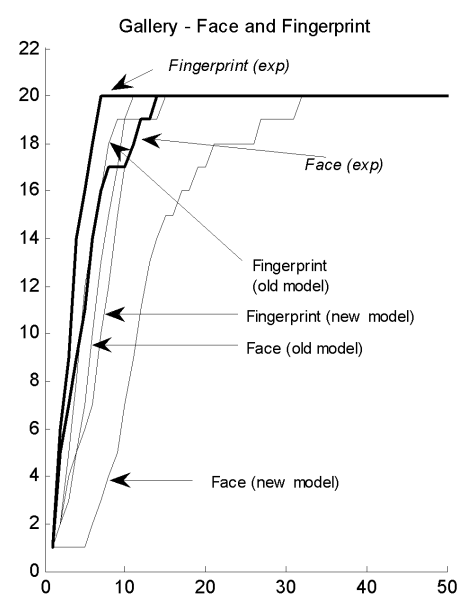

(a)

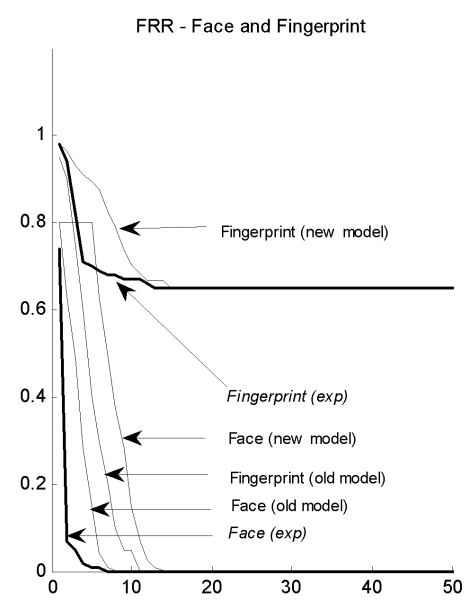

(b)

Fig. 3. Client n.11. Experimental trend and predicted values of gallery sizes (a) and FRR (b) for fingerprint and face biometrics. Values predicted by the previous model and the new proposed model are shown.

The following observations can be drawn from Figures 2-4.

The model is able to predict the experimental performance, with a negligible difference. Predictions are more accurate than those obtained in [10], due to improvements in the model. That model estimates FRR by neglecting the differences in 
expressive power between isolated and connected samples. Consequently, if a gallery reaches its maximum size thanks to the other biometric (co-update scenario), the previous model estimates $F R R=0$. However, in the real scenario, due to the presence of isolated samples, the true value of FRR will be greater than $0(\mathrm{FRR}=1$ if all the samples in gallery are isolated). As we can notice from Figs. 2-4, this drawback is completely eliminated in the present model.

The saturation of theoretical and experimental curves is obviously due to the form of eqs. $(5,7)$. In particular, the weight $w$ tends to decrease as the gallery size of both matchers increases, depending on the value of their FRR. Thus, the size of galleries must converge to $N_{T O T}$ if $m_{i}$ is not zero. According to the model, at the end of coupdate process, $\operatorname{FRR}\left(n=N_{T O T}\right)=f_{I}$. It is worth noting that the correct prediction of FRR values is matter of primary importance both for the validation of the model and for the designers, that need relevant information about the performance of the system.

Some issues have not yet been investigated: (1) in real environments $N_{T O T}$ is unknown. This problem can be overcame by considering $N_{T O T}$ as the number of samples in the batch exhibiting, for each client, a score above the zeroFAR threshold adopted; (2) the same for the estimation of $m_{i}$. These assumptions do not consider the problem of impostors insertion, a still open issue as remarked in [9, 10]. Therefore, in a future work, we will investigate the impact of $N_{T O T}$ and $m_{i}$ predicted on a batch of samples including even impostors, on a larger, non chimerical data set.

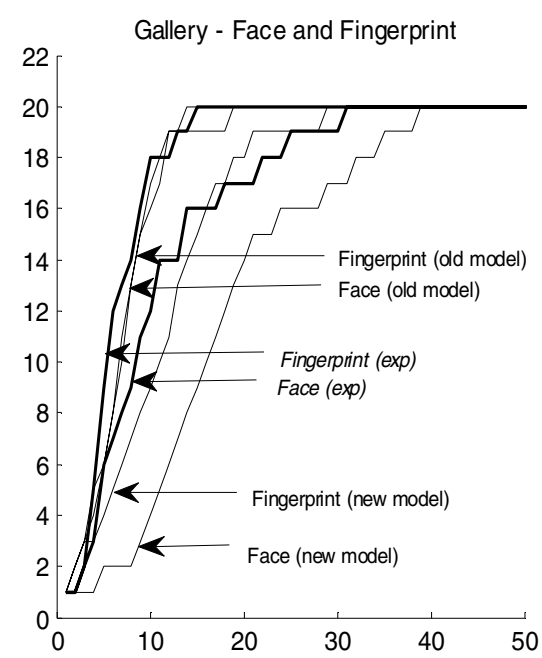

(a)

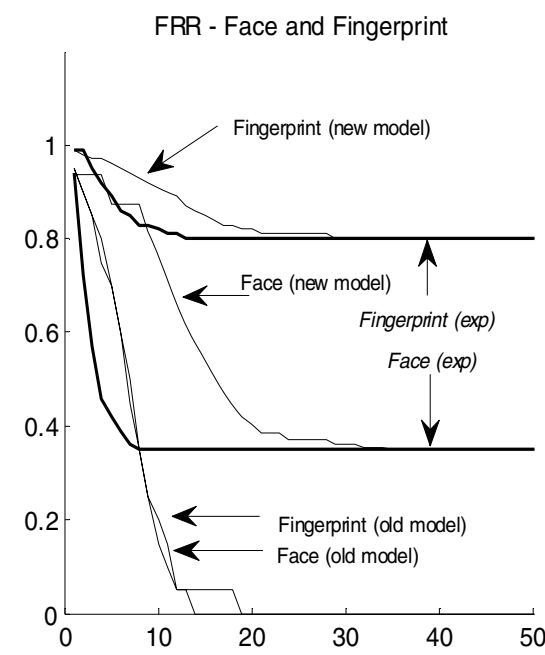

(b)

Fig. 4. Client n. 30. Experimental trend and predicted values of gallery sizes (a) and FRR (b) for fingerprint and face biometrics. Values predicted by the previous model and the new proposed model are shown. 


\section{Conclusions}

In this paper, we improved the theoretical framework explaining the template coupdate behaviour proposed in a state-of-the-art work, by a more effective and realistic modelling of the verification performance in terms of FRR. The model still assumes that no impostors are collected during system operation, by setting the updating threshold at zeroFAR. Therefore, the assessment of verification performance is only related to that operational point.

Although we pointed out some issues still remain, we believe that the template coupdate algorithm is well explained by proposed model. Therefore, it is suitable for designing bi-modal verification systems which can improve with use.

\section{References}

1. Li, S.Z., Jain, A.K. (eds.): Handbook of face recognition. Springer, Heidelberg (2005)

2. Maltoni, D., Maio, D., Jain, A.K., Prabhakar, S.: Handbook of fingerprint recognition. Springer, Heidelberg (2003)

3. Uludag, U., Ross, A., Jain, A.K.: Biometric template selection and update: a case study in fingerprints. Pattern Recognition 37(7), 1533-1542 (2004)

4. Jiang, X., Ser, W.: Online Fingerprint Template Improvement. IEEE Trans. PAMI 24(8), 1121-1126 (2002)

5. Ryu, C., Hakil, K., Jain, A.: Template adaptation based fingerprint verification. In: Proc. of ICPR, Hong Kong, vol. 4, pp. 582-585 (2006)

6. Roli, F., Marcialis, G.L.: Semi-supervised PCA-based face recognition using self-training. In: Yeung, D.-Y., Kwok, J.T., Fred, A., Roli, F., de Ridder, D. (eds.) SSPR 2006 and SPR 2006. LNCS, vol. 4109, pp. 560-568. Springer, Heidelberg (2006)

7. Roli, F., Didaci, L., Marcialis, G.L.: Template co-update in multimodal biometric systems. In: Lee, S.-W., Li, S.Z. (eds.) ICB 2007. LNCS, vol. 4642, pp. 1194-1202. Springer, Heidelberg (2007)

8. Roli, F., Didaci, L., Marcialis, G.L.: Adaptive biometric systems that can improve with use. In: Ratha, N., Govindaraju, V. (eds.) Advances in Biometrics: Sensors, Systems and Algorithms, pp. 447-471. Springer, Heidelberg (2008)

9. Rattani, A., Marcialis, G.L., Roli, F.: Capturing large intra-class variations of biometric data by template co-updating. In: IEEE Workshop on Biometrics, Int. Conf. on Vision and Pattern Recognition CVPR 2008, Anchorage, Alaska, USA (2008) (in press)

10. Didaci, L., Marcialis, G.L., Roli, F.: A Theoretical and Experimental Analysis of Template Co-update in Biometric Verification Systems. In: da Vitora-Lobo, N., Kasparis, T., Roli, F., Kwok, J.T., Georgiopoulos, M., Anagnostopoulos, G.C., Loog, M. (eds.) S+SSPR 2008. LNCS, vol. 5342, pp. 765-774. Springer, Heidelberg (2008)

11. Ross, A., Nandakumar, K., Jain, A.K.: Handbook of Multibiometrics. Springer, Heidelberg (2006)

12. http://www. equinoxsensors.com/products/HID.html

13. Jain, A.K., Hong, L., Bolle, R.: On-line Fingerprint Verification. IEEE Transactions on Pattern Analysis and Machine Intelligence 19(4), 302-314 (1997)

14. Turk, M., Pentland, A.: Eigenfaces for Face Recognition. Journal of Cognitive Neuroscience 3(1), 71-86 (1991) 